\title{
Neuroscience and the possibility of locally determined choices: Reply to Adina Roskies and Eddy Nahmias
}

\author{
Marcelo Fischborn \\ marcelofischborn@gmail.com \\ Universidade Federal de Santa Maria
}

\begin{abstract}
In a previous paper (Fischborn, 2016), I argued that neuroscience and psychology could in principle undermine libertarian free will by providing support for a subset of what I called 'statements of local determination'. I also argued that Libet-style experiments have not so far supported statements of that sort. In a commentary to the paper, Adina Roskies and Eddy Nahmias (2016) accept the claim about Libet-style experiments, but reject the claim about the possibilities of neuroscience. Here I explain why I still disagree with their conclusion, despite being sympathetic to a lot of what they say in support of it.

Keywords: free will; neuroscience; determinism; incompatibilism; choice
\end{abstract}

Note: The final version of this paper has been published in Philosophical Psychology and is available at: http://www.tandfonline.com/eprint/yw9kyzE5NwSYTiCDIYez/full

In a previous paper (Fischborn, 2016), I argued that neuroscience and psychology could in principle undermine libertarian free will by providing support for a subset of what I called 'statements of local determination'. I also argued that Libet-style experiments have not so far supported statements of that sort. In a commentary to the paper, Adina Roskies and Eddy Nahmias (2016) accept the claim about Libet-style experiments, but reject the claim about the possibilities of neuroscience. They argue that "neuroscience cannot establish the relevant kind of determinism” (p. 2) and that "in principle, neuroscience will not be able undermine libertarian free will” (p. 2). In this short reply, I explain why I remain unpersuaded by their argument. 
As I defined the notion, statements of local determination are statements of the following sort:

LD1. For any event $x$, and any subject $s$, if an $x$ that is a pattern of neural activity of type $B$ occurs in s's brain, then $s$ will decide to push a given button. (2016, p. 497)

I argued that LD1 entails that a choice resulting from the occurrence of a pattern of neural activity of type B is not free-if one is assuming an incompatibilist conception of free will such as Roderick Chisholm's (1964) — and that it is not an (undetermined) self-forming action —if one is assuming an incompatibilist conception of free will such as Robert Kane’s (1996). And I described how the truth of a sufficiently large number of statements of local determination similar to LD1 would reduce the plausibility of the claim that choices that are free in an incompatibilist sense exist. I argued that "sciences other than fundamental physics, such as neuroscience and psychology, could in principle support those deterministic statements.” (2016, p. 498).

Roskies and Nahmias reject what this last claim implies about neuroscience, and their argument relies on the possibilities that (1) mental states are realized by the brain in multiple ways (multiple-realizability), that (2) mental states are constituted by states beyond the brain (extended or embodied cognition), and that (3) neural networks are complex and chaotic (complexity/chaos). I would like to distinguish certain sorts of claims about what a specific science can do in order to show why I remain unpersuaded by their conclusion despite being sympathetic to a lot of what they say in support of it.

Regarding a hypothesis like LD1, we can distinguish the following claims:

(a) Neuroscience has supported LD1.

(b) Neuroscience can support LD1 if $\mathrm{X}$ is true (for some $\mathrm{X}$ ).

(c) Neuroscience can support LD1 if LD1 is true. 
Roskies, Nahmias, and I agree that (a) is false. We also agree that (b) is false if we substitute ' $\mathrm{X}$ ' for the thesis that minds extend beyond the brain, or the thesis that mental states can be realized in multiple ways. (I also agree that (b) may be false if we substitute ' $\mathrm{X}$ ' for the claim that neural networks are chaotic in an indeterministic way.) But I think (c) is true-and I am not sure Roskies and Nahmias need to disagree with this. As I understand it, (c) is similar to the claim that physics could show, for example, that time travel is possible. It can be true that physics could in principle show that time travel is possible even if time travel is actually physically impossible. Saying that physics can in principle show that time travel is possible (if time travel is actually possible) says nothing about the truth or plausibility of the possibility of time travel; it only says that physics is the right science for an investigation on the possibility of time travel. Moreover, (c) is not trivially true because, for example, the claim that "Sociology can support LD1 if LD1 is true” seems clearly false. Accordingly, the claim that neuroscience can in principle support LD1 is not meant to suggest anything about the truth or likelihood of LD1. By asserting (c), I am just assigning to neuroscience the task of assessing the truth or falsity of LD1.

I take the previous distinctions to be perfectly consistent with my original claim that neuroscience could in principle support a group of relevant statements of local determination. As I originally said, the very fact that LD1 purposely resembles the way Soon, Brass, Heize, and Heynes (2008) report their results suggests that statements like LD1 are a topic for neuroscience. And I added that the claim that neuroscience could in principle support statements like LD1 "is not meant to suggest that it would be easy to discover whether specific brain areas and patterns of neural activity in fact determine specific kinds of choices” (2016, p. 498). Roskies and Nahmias say that "local determination is unlikely to be established by neuroscience in any form that should trouble compatibilists or libertarians” (2016, p. 1). I agree with that and, as I explained above, I take this agreement to be consistent with the claim that neuroscience could in principle support local determination. The mere fact that local determination of the relevant sort is unlikely is not a threat to my point.

A second objection Roskies and Nahmias raise is that "local determination would not raise a challenge to free will without assuming universal determinism” (p. 5). In my original 
paper, I described how statements of local determination could impact libertarian theories of free will such as Chisholm's and Kane’s. Roskies and Nahmias note that such theorists endorse incompatibilism "only because they first develop or accept incompatibilist arguments using universal determinism” (p. 4). Therefore, they ask, "Why should we accept the incompatibilism that motivates these libertarians unless we are considering the sort of universal determinism that neuroscience cannot establish[?]” (p. 4). As Roskies and Nahmias properly note, I did not provide any argument for incompatibilism—and neither did I intend to do so. My aim was to examine whether the conditions for the existence of free will posited by some theories are ever satisfied, and in doing so I disregarded (at least for the moment) the question of why those theories arrived at conclusions about the incompatibility of free will and determinism. Thus, my claim that neuroscience could in principle undermine free will should be read conditionally: neuroscience could in principle undermine free will if free will requires that at least some choices are not determined by previous neural activity. For the theories I considered, part of the reason why such undetermined choices are required is that only some forms of indetermination leave unconditional room for alternative choices and actions - a point that has been influential in different ways in the history of incompatibilism.

Roskies and Nahmias also point out that in theories of free will such as Kane's free will can exist even if most of our choices are determined, provided that a few of them are not. On this basis, they argue that evidence for the claim that some choices are determined by previous neural activity would not give us reasons to doubt that free will (in the sense described by such theory) exists. In order to show that no choice is free in the sense considered, Roskies and Nahmais say, one would need "reasons to think that indeterministic events do not affect brain activity, and thus to think that global determinism is true” (p. 7). In the paper, I described DNC, a version of a more global form of determinism (still weaker than universal determinism) that I thought would undermine the existence of free will in Kane’s sense: 
DNC. For any subject $s$, any choice $x$, and any course of action $X$, if s chooses to do $X$, then there is a previous event $y$ of a type $Y$ in s's brain, such that whenever an event of type $Y$ occurs in someone's brain, then this subject will choose for the course of action $X$. (2016, p. 498)

If we had reasons to think that DNC is true, I think we would have reasons to think that no choice is free in the sense postulated by Kane. Nahmias and Roskies certainly doubt that neuroscience will ever show that DNC is true; me too. But, no matter how unlikely DNC sounds given what we currently know about brains and decisions, I still believe that, if DNC is true, then neuroscience could in principle show that it is, and, therefore, that neuroscience can in principle undermine the existence of free will in certain incompatibilist senses.

\section{References}

Chisholm, R. (1964). "Human freedom and the self”. In: Pereboom, D. (Ed.), Free will, pp. 172184. Indianapolis: Hackett.

Fischborn, M. (2016). "Libet-style experiments, neuroscience, and libertarian free will”, Philosophical Psychology 29: 494-502.

Kane, R. (1996). The significance of free will. Oxford: Oxford University Press.

Roskies, A. and Nahmias, E. (2016). ““'Local determination”, even if we could find it, does not challenge free will: Commentary on Marcelo Fischborn”, Philosophical Psychology : 1-9.

Soon, C. S.; Brass, M.; Heinze, H.-J. and Haynes, J.-D. (2008). "Unconscious determinants of free decisions in the human brain”, Nature Neuroscience 11: 543-545. 